\title{
Synchronization of Time Delayed Fractional Order Chaotic Financial System
}

\author{
Weiwei Zhang, ${ }^{1}$ Jinde Cao, ${ }^{2,3}$ Ahmed Alsaedi, ${ }^{3}$ and Fuad Eid S. Alsaadi ${ }^{4}$ \\ ${ }^{1}$ School of Mathematics and Computational Science, Anqing Normal University, Anqing 246011, China \\ ${ }^{2}$ School of Mathematics, Southeast University, Nanjing 210096, China \\ ${ }^{3}$ Department of Mathematics, Faculty of Science, King Abdulaziz University, Jeddah 21589, Saudi Arabia \\ ${ }^{4}$ Department of Electrical and Computer Engineering, King Abdulaziz University, Jeddah 21589, Saudi Arabia \\ Correspondence should be addressed to Weiwei Zhang; wwzhahu@aliyun.com
}

Received 3 March 2017; Accepted 23 August 2017; Published 1 October 2017

Academic Editor: Gian I. Bischi

Copyright (c) 2017 Weiwei Zhang et al. This is an open access article distributed under the Creative Commons Attribution License, which permits unrestricted use, distribution, and reproduction in any medium, provided the original work is properly cited.

\begin{abstract}
The research on a time delayed fractional order financial chaotic system is a hot issue. In this paper, synchronization of time delayed fractional order financial chaotic system is studied. Based on comparison principle of linear fractional equation with delay, by using a fractional order inequality, a sufficient condition is obtained to guarantee the synchronization of master-slave systems. An example is exploited to show the feasibility of the theoretical results.
\end{abstract}

\section{Introduction}

In micro-macroeconomics, the study on the dynamics of financial and economical systems is an interesting and important topic [1]. The features of economic data were presented in view of the dynamical behaviors of systems. Many nonlinear continuous models have been introduced to study complex economic dynamics, such as the IS-LM model [2], Goodwin's accelerate model [3], the forced Vander-Pol model [4], and Behrens-Feichtinger model [5]. And the same as the other systems in world, financial system, as a nonlinear system, displays many complex dynamical behaviors, such as depending on initial value sensitivity, the complex phase portraits, positive Lyapunov exponents, and fractal properties. Chaotic phenomena in financial systems mean the systems will have inherent indefiniteness; it is difficult to make effective decision-making by makers, which threats the safety of investment. Therefore, to study the dynamical behaviors in economical and financial systems is indispensable.

Compared with classical integer calculus, the merit of fractional calculus is that it provides an excellent instrument for the description of memory and hereditary properties of dynamical processes [6-8]. Meanwhile, the financial variables such as interest rates, stock market prices, and foreign exchange rates possess long memories, which make it more appropriate to use fractional models compared with integer order ones in financial systems [9-12]. Furthermore, the author found that there existed many attractors in fractional order financial systems, such as fixed points, limit cycles, periodic motions, and chaotic attractors [9].

It is known that time delay can affect oscillation and instability behavior of dynamical systems. Time delay means that the policy from being made to taking effect will have to need some time in financial system, and its influence cannot be neglected. It dominates the decision which makes the policy intervene the economy. Since the pioneering work [13] that time delay was introduced to economic dynamics, the research on the delayed financial system has become one of the hot issues which has received more attention [14-17]. The synchronization of fractional order financial chaotic system with time delay is worth discussing. Recently, there are some works about synchronization on fractional order financial system without delay [18-21]; for example, synchronization and antisynchronization of fractional chaotic financial system via active control strategy were investigated in [18]; control and synchronization of fractional order financial system based on linear control were studied in [19]. However, the problem of synchronization for fractional order chaotic 
financial system with time delay has not been investigated in the literature.

Given the above discussions, in this paper, based on comparison principle of linear fractional equation with delay, by applying a fractional inequality, a sufficient condition is achieved to ensure the synchronization of fractional order time delayed chaotic financial systems. The result is simple and extremely effective.

The remainder of this paper is organized as follows. In Section 2, some necessary definitions and useful lemmas are introduced, and the model description is given. In Section 3, the synchronization schemes are presented, and sufficient conditions for synchronization are obtained. Numerical simulations are presented in Section 4. Some conclusions are drawn in Section 5.

\section{Preliminaries and Model Description}

There are some definitions of the fractional order derivatives. Riemann-Liouville fractional derivative and Caputo fractional derivative are mostly used. The main advantage of the Caputo derivative is that its Laplace transform only requires integer order derivatives for the initial conditions; the definition of Caputo derivative is given in this paper.

Definition 1 (see [6]). The fractional integral with noninteger order $\alpha>0$ of function $x(t)$ is defined as follows:

$$
I^{\alpha} x(t)=\frac{1}{\Gamma(\alpha)} \int_{t_{0}}^{t}(t-\tau)^{\alpha-1} x(\tau) d \tau,
$$

where $\Gamma(\cdot)$ is the Gamma function and $\Gamma(s)=\int_{0}^{\infty} t^{s-1} e^{-t} d t$.

Definition 2 (see [6]). The Caputo derivative of fractional order $\alpha$ of function $x(t)$ is defined as follows:

$$
D^{\alpha} x(t)=\frac{1}{\Gamma(n-\alpha)} \int_{t_{0}}^{t}(t-\tau)^{n-\alpha-1} x^{(n)}(\tau) d \tau,
$$

where $n-1<\alpha<n \in Z^{+}$.

In this paper, we consider a fractional order financial system with time delay, which is described by

$$
\begin{aligned}
& D^{\alpha} x(t)=z(t)+(y(t-\tau)-a) x(t), \\
& D^{\alpha} y(t)=1-b y(t)-x^{2}(t-\tau), \\
& D^{\alpha} z(t)=-x(t-\tau)-c z(t),
\end{aligned}
$$

where $0<\alpha<1, x, y, z$ are three state variables, $x$ stands for the interest rate, $y$ represents the investment demand, $z$ denotes the price index, $a$ is the saving amount, $b$ is the cost per investment, $c$ is the elasticity of demand of the commercial markets and parameters, $a, b, c$ are nonnegative real constants, and $\tau>0$ is time delay of the system. When $\alpha=0.9, a=3, b=0.1, c=1$, and $\tau=0.1$, system (3) displays chaotic attractors with the initial value $x(0)=0.1, y(0)=4$, and $z(0)=0.5$, which is shown in Figure 1 .

In order to get main results, we give some lemmas as follows.
Lemma 3 (see [22]). Suppose $x(t) \in R^{n}$ is a continuous and differentiable vector-value function. Then, for any time instant $t \geq t_{0}$, we have

$$
\frac{1}{2} D^{\alpha} x^{T}(t) x(t) \leq x^{T}(t) D^{\alpha} x(t)
$$

where $0<\alpha<1$.

Lemma 4 (see [23]). Suppose $V(t) \in R^{1}$ is a continuously differentiable and nonnegative function, satisfying

$$
\begin{aligned}
D^{\alpha} V(t) & \leq-a V(t)+b V(t-\tau), \quad 0<\alpha<1 \\
V(t) & =\varphi(t) \geq 0, \quad t \in[-\tau, 0],
\end{aligned}
$$

where $t \in[0,+\infty)$. If $a>b>0$, for all $\varphi(t) \geq 0, \tau>0$, then $\lim _{t \rightarrow+\infty} V(t)=0$.

\section{Main Results}

In this section, we discuss the synchronization of fractional order delayed financial system. The main aim is to design a proper controller to achieve synchronization between master system and slave system. Without loss of generally, the master system is chosen as

$$
\begin{aligned}
& D^{\alpha} x_{m}(t)=z_{m}(t)+\left(y_{m}(t-\tau)-a\right) x_{m}(t), \\
& D^{\alpha} y_{m}(t)=1-b y_{m}(t)-x_{m}^{2}(t-\tau), \\
& D^{\alpha} z_{m}(t)=-x_{m}(t-\tau)-c z_{m}(t) .
\end{aligned}
$$

The slave system is selected as

$$
\begin{aligned}
& D^{\alpha} x_{s}(t)=z_{s}(t)+\left(y_{s}(t-\tau)-a\right) x_{s}(t)+u_{1}(t), \\
& D^{\alpha} y_{s}(t)=1-b y_{s}(t)-x_{s}^{2}(t-\tau)+u_{2}(t), \\
& D^{\alpha} z_{s}(t)=-x_{s}(t-\tau)-c z_{s}(t)+u_{3}(t)
\end{aligned}
$$

where $u_{i}(t)(i=1,2,3)$ are the external control inputs to be designed.

Let $e_{1}(t)=x_{s}-x_{m}, e_{2}(t)=y_{s}-y_{m}$, and $e_{3}(t)=z_{s}-z_{m}$ be the synchronization errors.

Subtracting the master system (4) from the slave system (5), the error system is obtained as follows:

$$
\begin{aligned}
D^{\alpha} e_{1}(t)= & e_{3}(t)+e_{2}(t-\tau) x_{s}(t)+y_{m}(t-\tau) e_{1} \\
& -a e_{1}(t)+u_{1}(t) \\
D^{\alpha} e_{2}(t)= & -b e_{2}(t)-e_{1}(t-\tau)\left(x_{s}(t-\tau)+x_{m}(t-\tau)\right) \\
& +u_{2}(t) \\
D^{\alpha} e_{3}(t)= & -e_{1}(t-\tau)-c e_{3}(t)+u_{3}(t) .
\end{aligned}
$$



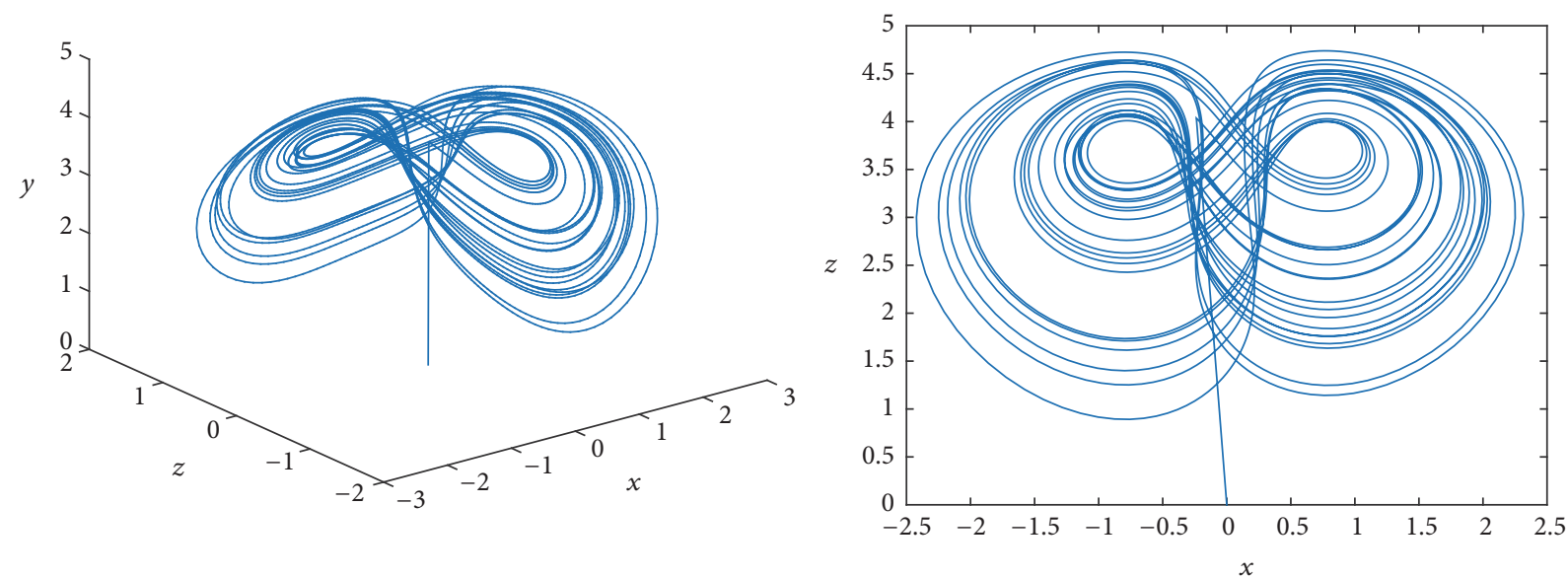

FIGURE 1: Chaotic attractors of system (3).

Control schemes $u_{i}(t)(i=1,2,3)$ are defined as follows:

$$
\begin{aligned}
& u_{1}(t)=-e_{2}(t-\tau) x_{s}(t)-y_{m}(t-\tau) e_{1}(t)+k_{1} e_{1}(t), \\
& u_{2}(t)=e_{1}(t-\tau)\left(x_{s}(t-\tau)+x_{m}(t-\tau)\right)+k_{2} e_{2}(t), \\
& u_{3}(t)=k_{3} e_{3}(t),
\end{aligned}
$$

where $k_{1}, k_{2}, k_{3}$ are feedback gains.

Combining (8) with (9), the error system is given by

$$
\begin{aligned}
& D^{\alpha} e_{1}(t)=\left(-a+k_{1}\right) e_{1}(t)+e_{3}(t), \\
& D^{\alpha} e_{2}(t)=\left(-b+k_{2}\right) e_{2}(t), \\
& D^{\alpha} e_{3}(t)=\left(-c+k_{3}\right) e_{3}(t)-e_{1}(t-\tau) .
\end{aligned}
$$

Theorem 5. If the feedback gains $k_{1}, k_{2}, k_{3}$ satisfy the following condition: $l>1$, the synchronization between system (6) and system (7) is achieved, where $l=\min \left\{a-k_{1}-1 / 2, b-k_{2}, c-k_{3}-\right.$ $1\}$ denotes the minimal value of $\left\{a-k_{1}-1 / 2, b-k_{2}, c-k_{3}-1\right\}$.

Proof. Construct a Lyapunov function:

$$
V(t)=\frac{1}{2}\left(e_{1}^{2}(t)+e_{2}^{2}(t)+e_{3}^{2}(t)\right) .
$$

From Lemma 3,

$$
\begin{aligned}
D^{\alpha} V(t)= & D^{\alpha}\left[\frac{1}{2}\left(e_{1}^{2}(t)+e_{2}^{2}(t)+e_{3}^{2}(t)\right)\right] \\
\leq & e_{1}(t) D^{\alpha} e_{1}(t)+e_{2}(t) D^{\alpha} e_{2}(t) \\
& +e_{3}(t) D^{\alpha} e_{3}(t) \\
= & e_{1}(t)\left[\left(-a+k_{1}\right) e_{1}(t)+e_{3}(t)\right] \\
& +e_{2}(t)\left[\left(-b+k_{2}\right) e_{2}(t)\right] \\
& +e_{3}(t)\left[\left(-c+k_{3}\right) e_{3}(t)-e_{1}(t-\tau)\right] \\
\leq & e_{1}(t)\left(-a+k_{1}\right) e_{1}(t)+\left|e_{1}(t)\right|\left|e_{3}(t)\right| \\
& +e_{2}(t)\left(-b+k_{2}\right) e_{2}(t) \\
& +e_{3}(t)\left(-c+k_{3}\right) e_{3}(t)+\left|e_{3}(t)\right|\left|e_{1}(t-\tau)\right|
\end{aligned}
$$

$$
\begin{aligned}
\leq & \left(-a+k_{1}\right) e_{1}^{2}(t)+\frac{1}{2}\left(e_{1}^{2}(t)+e_{3}^{2}(t)\right) \\
& +\left(-b+k_{2}\right) e_{2}^{2}(t)+\left(-c+k_{3}\right) e_{3}^{2}(t) \\
& +\frac{1}{2}\left(e_{3}^{2}(t)+e_{1}^{2}(t-\tau)\right) \\
= & \left(-a+k_{1}+\frac{1}{2}\right) e_{1}^{2}(t)+\left(-b+k_{2}\right) e_{2}^{2}(t) \\
& +\left(-c+k_{3}+1\right) e_{3}^{2}(t)+\frac{1}{2} e_{1}^{2}(t-\tau) \\
\leq & -2 l V(t)+2 V(t-\tau) .
\end{aligned}
$$

According to Lemma 4, when $l>1$, then system (6) synchronizes system (7).

Remark 6. Recently, there are a few works about the synchronization of fractional order chaotic financial systems [18-21], but these results are without considering time delay. Here, we consider a fractional order delayed chaotic financial systems.

Remark 7. Compared with $[18,19]$, in this paper, comparison principle of linear fractional equation with delay is used, and the sufficient condition of the synchronization between master-slave systems is achieved; from Figures 3-5, the controllers are effective.

\section{Numerical Simulations}

In this section, the numerical simulations are given to show the effectiveness of the theoretical results. The step-length $h=$ 0.01 in the Adams-Bashforth-Moulton predictor-corrector scheme is taken [24]. The parameters of the time delayed fractional order financial chaotic system are chosen as $\alpha=$ $0.9, a=3, b=0.1, c=1$, and $\tau=0.1$. The initial values of the master system and slave system are selected as $x_{m}(0)=2, y_{m}(0)=1, z_{m}(0)=4, x_{s}(0)=0.2, y_{s}(0)=0.3$, and $z_{\mathrm{s}}(0)=0.1$, respectively. The synchronization errors are 


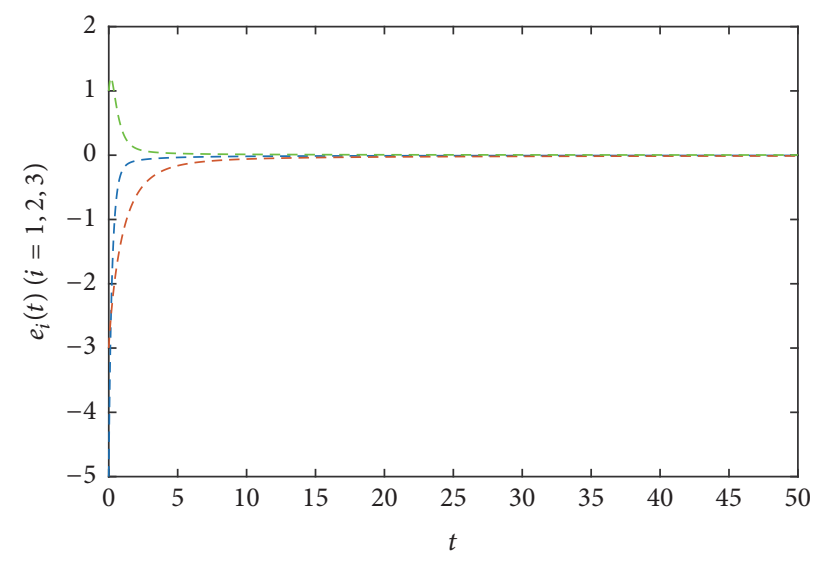

FIGURE 2: The errors state of $e_{1}, e_{2}, e_{3}$.

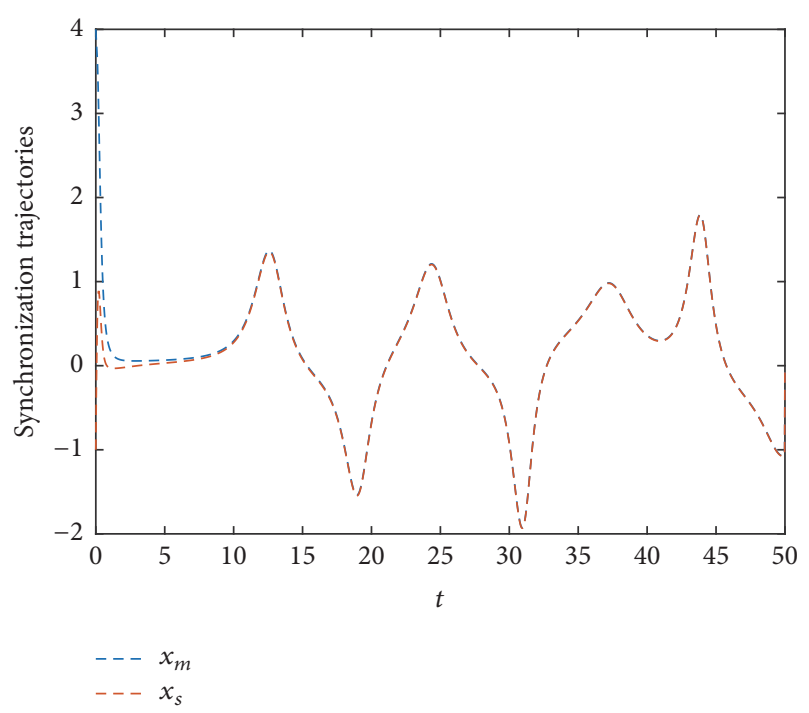

FIgURE 3: The synchronization trajectories of $x_{m}, x_{s}$.

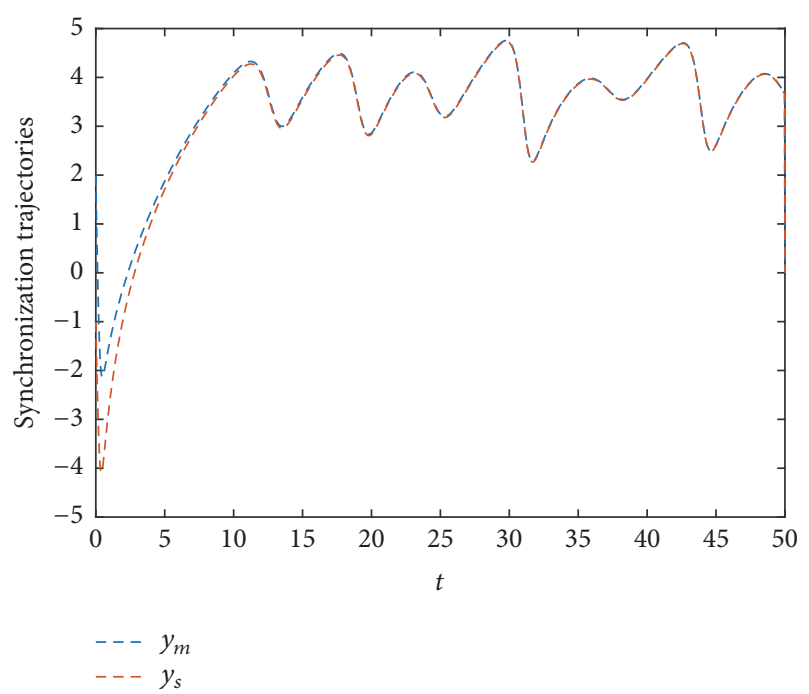

FIGURE 4: The synchronization trajectories of $y_{m}, y_{s}$.

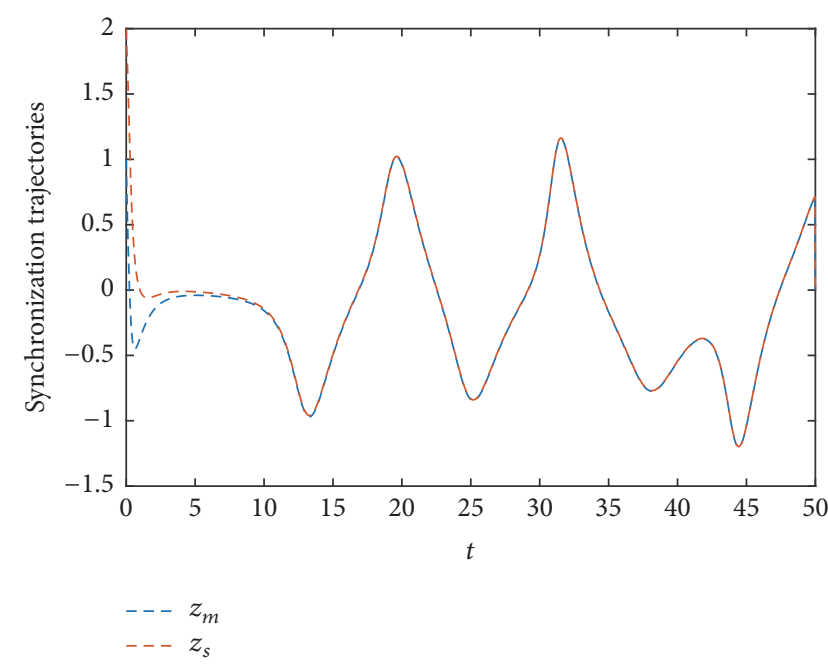

FIGURE 5: The synchronization trajectories of $z_{m}, z_{s}$.

depicted in Figure 2. The state synchronization trajectories of the master-slave systems are shown in Figures 3-5.

\section{Conclusions}

Time delay is a sensitive factor for the financial system. In this paper, we study the synchronization of a generalized financial system which takes time delay into consideration. The sufficient condition for synchronization is established according to comparison principle of linear fractional equation with delay. The numerical simulations indicate that the method designed for synchronization is effective.

\section{Conflicts of Interest}

The authors declare no conflicts of interest.

\section{Acknowledgments}

This work was supported by the National Natural Science Foundation of China (Grant no. 11571016), the Natural Science Foundation of Anhui Province (Grant no. 1608085MA14), and the Natural Science Foundation of the Higher Education Institutions of Anhui Province (Grant no. KJ2015A152).

\section{References}

[1] R. Shone, Economic Dynamics: Phase Diagrams and their Economic Application, Cambridge University Press, Cambridge, UK, 2nd edition, 2002.

[2] L. De Cesare and M. Sportelli, "A dynamic IS-LM model with delayed taxation revenues," Chaos, Solitons and Fractals, vol. 25, no. 1, pp. 233-244, 2005.

[3] H.-W. Lorenz and H. E. Nusse, "Chaotic attractors, chaotic saddles, and fractal basin boundaries: Goodwin's nonlinear accelerator model reconsidered," Chaos, Solitons and Fractals, vol. 13, no. 5, pp. 957-965, 2002. 
[4] A. C. Chian, E. L. Rempel, and C. Rogers, "Complex economic dynamics: chaotic saddle, crisis and intermittency," Chaos, Solitons and Fractals, vol. 29, no. 5, pp. 1194-1218, 2006.

[5] G. Feichtinger, Economic Evolution and Demographic Change, vol. 395, Springer, Berlin, Germany, 1992.

[6] I. Podlubny, Fractional Differential Equations, vol. 198 of Mathematics in Science and Engineering, Academic Press, San Diego, Calif, USA, 1999.

[7] R. Hilfer, Applications of Fractional Calculus in Physics, World Scientific, Singapore, 2000.

[8] P. L. Butzer and U. Westphal, "An introduction to fractional calculus," in Applications of Fractional Calculus in Physics, pp. 1-85, World Scientific, Singapore, 2000.

[9] W. C. Chen, "Nonlinear dynamics and chaos in a fractionalorder financial system," Chaos, Solitons and Fractals, vol. 36, no. 5, pp. 1305-1314, 2008.

[10] M.-F. Danca, R. Garrappa, W. K. Tang, and G. Chen, "Sustaining stable dynamics of a fractional-order chaotic financial system by parameter switching," Computers and Mathematics with Applications, vol. 66, no. 5, pp. 702-716, 2013.

[11] I. Pan, S. Das, and S. Das, "Multi-objective active control policy design for commensurate and incommensurate fractional order chaotic financial systems," Applied Mathematical Modelling. Simulation and Computation for Engineering and Environmental Systems, vol. 39, no. 2, pp. 500-514, 2015.

[12] B. Xin and J. Zhang, "Finite-time stabilizing a fractional-order chaotic financial system with market confidence," Nonlinear Dynamics. An International Journal of Nonlinear Dynamics and Chaos in Engineering Systems, vol. 79, no. 2, pp. 1399-1409, 2015.

[13] M. Kalecki, "A macrodynamic theory of business cycles," Econometrica, vol. 3, no. 3, pp. 327-344, 1935.

[14] Z. Wang, X. Huang, and G. Shi, "Analysis of nonlinear dynamics and chaos in a fractional order financial system with time delay," Computers and Mathematics with Applications. An International Journal, vol. 62, no. 3, pp. 1531-1539, 2011.

[15] L. Zhou and Y. Li, "A generalized dynamic IS-LM model with delayed time in investment processes," Applied Mathematics and Computation, vol. 196, no. 2, pp. 774-781, 2008.

[16] A. Kaddar and H. Talibi Alaoui, "On the dynamic behavior of a delayed IS-LM business cycle model," Applied Mathematical Sciences, vol. 2, no. 29-32, pp. 1529-1539, 2008.

[17] A. Matsumoto and F. Szidarovszky, "Delayed dynamics in heterogeneous competition with product differentiation," Nonlinear Analysis. Real World Applications. An International Multidisciplinary Journal, vol. 11, no. 2, pp. 601-611, 2010.

[18] C. Huang and J. Cao, "Active control strategy for synchronization and anti-synchronization of a fractional chaotic financial system," Physica A. Statistical Mechanics and its Applications, vol. 473, pp. 262-275, 2017.

[19] L. Chen, Y. Chai, and R. Wu, "Control and synchronization of fractional-order financial system based on linear control," Discrete Dynamics in Nature and Society. An International Multidisciplinary Research and Review Journal, Art. ID 958393, 21 pages, 2011.

[20] Y. Xu and Z. He, "Synchronization of variable-order fractional financial system via active control method," Central European Journal of Physics, vol. 11, no. 6, pp. 824-835, 2013.

[21] M. Yang, D. Dong, and S. Ma, "Chaos control and projective synchronization of a fractional-order financial system," in Proceedings of the 2015 2nd International Conference on Information Science and Control Engineering, ICISCE 2015, pp. 649-653, chn, April 2015.
[22] N. Aguila-Camacho, M. A. Duarte-Mermoud, and J. A. Gallegos, "Lyapunov functions for fractional order systems," Communications in Nonlinear Science and Numerical Simulation, vol. 19, no. 9, pp. 2951-2957, 2014.

[23] S. Liang, R. Wu, and L. Chen, "Adaptive pinning synchronization in fractional-order uncertain complex dynamical networks with delay," Physica A. Statistical Mechanics and its Applications, vol. 444, pp. 49-62, 2016.

[24] K. Diethelm, N. J. Ford, and A. D. Freed, "A predictor-corrector approach for the numerical solution of fractional differential equations," Nonlinear Dynamics. An International Journal of Nonlinear Dynamics and Chaos in Engineering Systems, vol. 29, no. 1-4, pp. 3-22, 2002. 


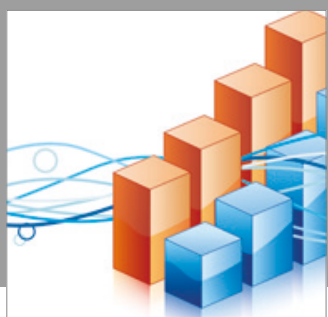

Advances in

Operations Research

vatersals

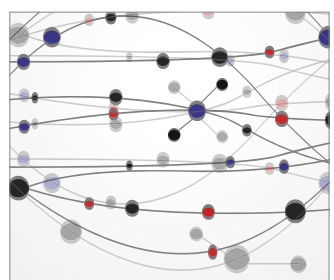

\section{The Scientific} World Journal
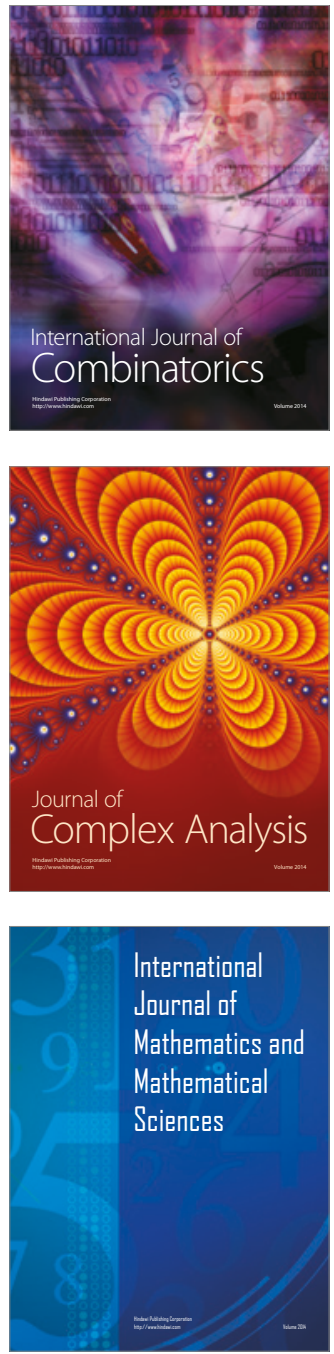
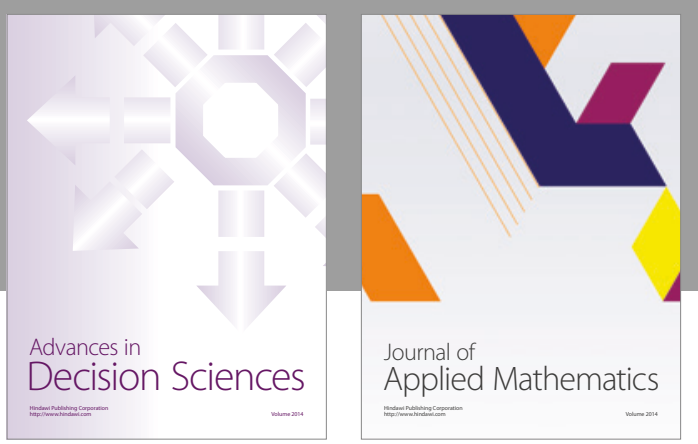

Algebra

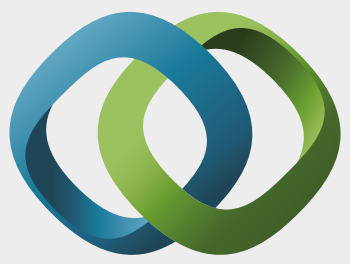

\section{Hindawi}

Submit your manuscripts at

https://www.hindawi.com
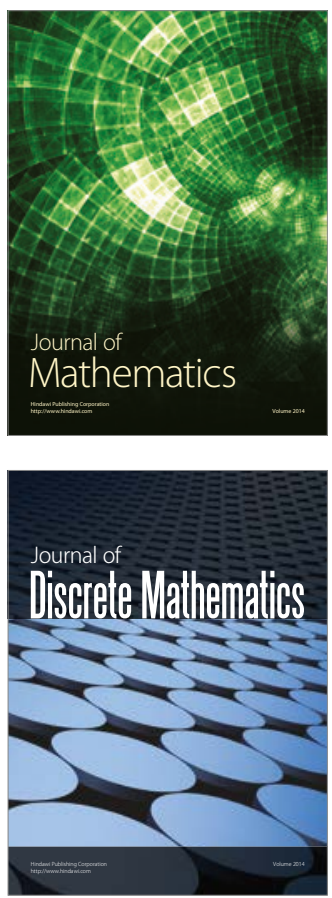

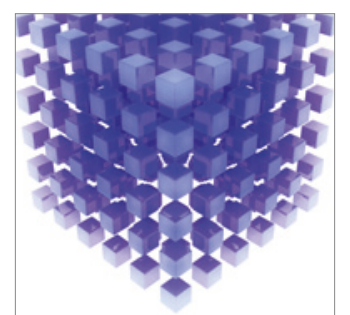

Mathematical Problems in Engineering
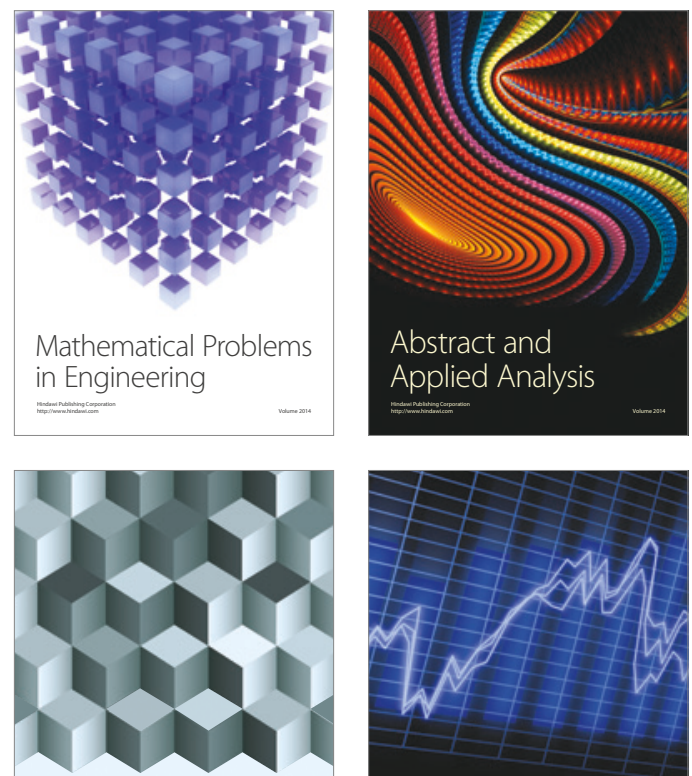

Journal of

Function Spaces

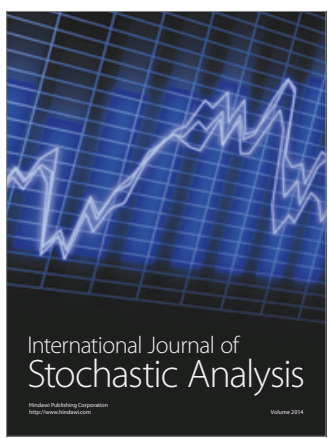

Probability and Statistics
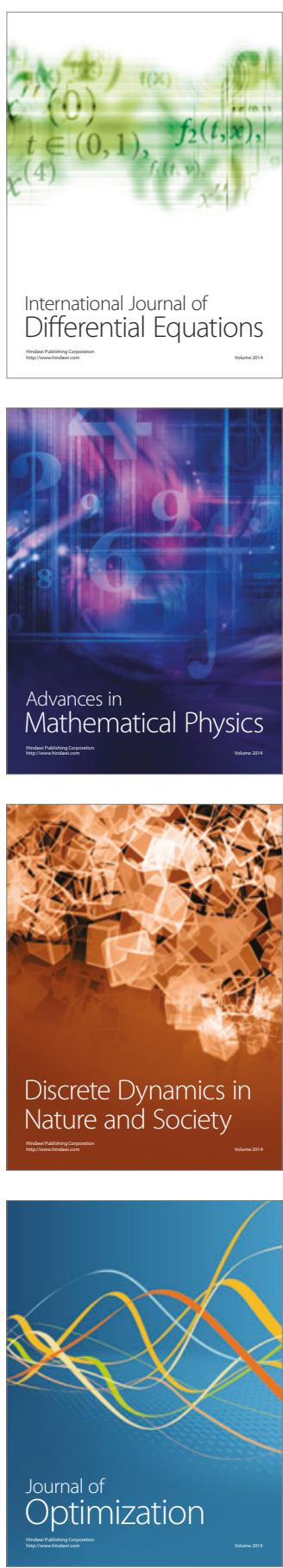